\title{
Meningitis due to Gemella haemolysans after radiofrequency trigeminal rhizotomy
}

\author{
RG MITCHELL, PJ TEDDY \\ From the Department of Microbiology, John Radcliffe Hospital, and the Department of Neurological Surgery, \\ Radcliffe Infurmary, Oxford
}

SUMMARY Meningitis due to Gemella haemolysans developed in a 73 year old woman after thermolysis of the Gasserian ganglion for trigeminal neuralgia. The taxonomy of this organism is discussed, and previous cases of infection are reviewed.

Gemella haemolysans (formerly Neisseria haemolysans) is a commensal of the upper respiratory tract which has received little recognition by clinical bacteriologists and is seldom recognised as a cause of infection. We describe here a case of meningitis due to $G$ haemolysans which followed radiofrequency rhizotomy of the Gasserian ganglion.

\section{Case report}

A 73 year old woman suffered from right sided trigeminal neuralgia for one year, obtaining only partial relief from carbamazepine (Tegretol). Her respiratory tract appeared healthy, and radiographs of the paranasal sinuses and axial views of the skull and postnasal space were normal. Radiofrequency rhizotomy of the second and third divisions of the right trigeminal nerve was carried out.

In this operation, which is performed under a suitable neuroleptoanalgesia, the lower face is prepared with a solution of Hibitane in spirit. The cheek is infiltrated with $1 \%$ Xylocaine solution, and then a sterile needle, insulated along its shaft for all but the terminal $7 \mathrm{~mm}$, is passed through the cheek to the base of the skull. Entry into the foramen ovale is confirmed on radiograph and by showing an efflux of cerebrospinal fluid (CSF) through the needle hub. A stimulating current is passed through the needle to the trigeminal sensory root using a radiofrequency lesion generator, the appropriate division in which the pain was occurring is identified, and a thermal lesion made at this location. The needle is then withdrawn. Total operating time is generally $1 \mathrm{~h}$. In this particular patient, anatomical localisation proved difficult, and leakage of CSF occurred

Accepted for publication 8 January 1985 throughout the procedure, although it produced the intended analgesic result.

The day after operation the patient was free from pain but felt generally unwell, with a fever of $38^{\circ} \mathrm{C}$. On the second postoperative day she developed right sided headache and neck stiffness. Meningitis was diagnosed and a lumbar puncture was performed. The CSF contained 11000 neutrophil polymorphonuclear leucocytes $\times 10^{6} / 1$, with a protein concentration of $3.3 \mathrm{~g} / 1$ and a reduced glucose content. A Gram stained film of the CSF deposit showed numerous Gram negative diplococci, mostly intracellular, and a provisional diagnosis of meningococcal meningitis was made.

Because of a history of penicillin allergy, the patient was treated with chloramphenicol $0.6 \mathrm{~g}$ every $6 \mathrm{~h}$ and sulphadiazine $1 \mathrm{~g}$ every $6 \mathrm{~h}$, both given intravenously for nine days; this was followed by chloramphenicol $0.5 \mathrm{~g}$ every $6 \mathrm{~h}$ orally for a further five days. She responded well to treatment, although diminishing fever persisted until the eighth postoperative day. Three further specimens of CSF taken during the course of treatment showed only a moderate lymphocytosis and were sterile on culture.

\section{BACTERIOLOGY}

Culture of the CSF deposit produced a scanty growth of small greyish colonies on horse blood agar after incubation at $37^{\circ} \mathrm{C}$ for $48 \mathrm{~h}$ both aerobically, with or without additional $\mathrm{CO}_{2}$, and anaerobically. The colonies were surrounded by a definite zone of $\alpha$-haemolysis. On subculture, growth at $48 \mathrm{~h}$ was profuse at $22^{\circ} \mathrm{C}, 30^{\circ} \mathrm{C}$, and $42^{\circ} \mathrm{C}$. Growth did not occur on MacConkey's medium. Gram stain of a broth culture showed mainly Gram negative cocci which were single, in pairs with flattening of the adjacent surfaces, or in short chains. In addition, there were larger single cocci which were undoubtedly 
Gram positive.

The organism was non-motile, catalase negative, and oxidase negative (weak positive reaction with Kovacs' reagent at $60 \mathrm{~s}$ ). Growth did not occur in Hugh and Leifson's $0-F$ medium at $48 \mathrm{~h}$. The organism failed to survive heating to $60^{\circ} \mathrm{C}$ for $30 \mathrm{~min}$. The following reactions were obtained using the API Staph system (API Laboratory Products Limited) after incubation for $48 \mathrm{~h}$ : nitrate reduction negative; acetyl-methyl-carbinol production positive; arginine dihydrolase negative; urease negative; acid produced from glucose, maltose, fructose, sucrose, and mannose; no acid from lactose, trehalose, mannitol, xylitol, raffinose, or xylose. The organism was sensitive to penicillin, sulphonamide, and chloramphenicol by the disc agar plate method. These properties corresponded closely to the description of $G$ haemolysans given in Bergey's manual, ${ }^{1}$ our strain differing only in its pronounced $\alpha$-haemolysis on horse blood agar. The organism was excluded as Aerococcus viridans by Dr G Colman and confirmed as $G$ haemolysans by the National Collection of Type Cultures, Colindale.

\section{Discussion}

In 1938, Thjøtta and Bøe described a haemolytic species of Neisseria Trevisan, which they named Neisseria haemolysans. ${ }^{2}$ It was biochemically similar to $N$ sicca, but antigenically distinct. Berger and Wezel $^{3}$ found that this organism was less readily decolorised in the Gram stain than were other neisseriae. Berger ${ }^{4}$ further showed that it was catalase negative, oxidase negative, and attacked carbohydrates fermentatively, unlike neisseriae; he considered that it should be allocated to a new genus Gemella ("little twin") within the family Neisseriaceae, with a single species Gemella haemolysans. This view was supported by gas chromatographic studies, which showed that the fatty acid and sugar composition of $N$ haemolysans ATCC 10379 differed from that of other neisseriae studied. ${ }^{5}$ Reyn et $a l^{6}$ showed by electron microscopy that the cell wall structure, internal structures, and mode of division of $N$ haemolysans ATCC 10379 were typical of Gram positive cocci. The cell walls were comparatively thin and of varying thickness, which accounts for the variability of the Gram reaction. The DNA base ratio of the organism was 33.5 mean moles per cent, which is considerably lower than that of neisseriae but similar to that of the Streptococcaceae. On these grounds, Reyn et $a^{6}$ recommended that Gemella should be removed from the family Neisseriaceae and included as a new genus within the Streptococcaceae. This classification was accepted in the 8th edition of Bergey's manual,' in which a full description of the organism appears. Subsequently, Wilkinson and Jones, ${ }^{7}$ in a numerical taxonomic study of Listeria and related bacteria, obtained results which agreed with this classification, but considered finally that Gemella should be included with Listeria, Erysipelothrix, Lactobacillus, and Streptococcus in the family Lactobacillaceae.

Recent studies have shown that the peptidoglycan of $G$ haemolysans is biochemically identical to that of various other Gram positive organisms, such as streptococci and lactobacilli. ${ }^{8}$ Also, 16S rRNA analysis has shown that $G$ haemolysans is phylogenetically related to those Gram positive bacteria with low GC percent content, including Clostridium, Peptococcus, Streptococcus, Staphylococcus, Lactobacillus, and Kurthia.$^{8}$ The physiological properties of $G$ haemolysans are indistinguishable from those of Streptococcus morbillorum."

Thjøtta and $B ø \mathrm{e}^{2}$ isolated their strain from the sputum of a patient with chronic bronchitis, and the organism is considered to be a commensal of the oropharynx and respiratory tract.' 'One of the strains of $G$ haemolysans studied by Reyn et al ${ }^{6}$ was isolated from a child's blood culture, which also yielded a viridans streptococcus. Infections caused by this organism were unknown until 1978-9, when three cases of endocarditis due to $G$ haemolysans were diagnosed in three different hospitals in France, the strains being identified subsequently by the Pasteur Institute, Paris. ${ }^{10}$ All three patients had pre-existing dental sepsis. One of the patients lapsed into coma; the CSF showed pleocytosis but was sterile on culture. Because the organisms isolated from blood cultures were Gram variable, catalase negative, oxidase negative, and had the typical antibiotic sensitivity pattern of a streptococcus they were initially sent to a streptococcus reference laboratory for identification. The strains showed $\beta$-haemolysis only on certain media containing rabbit blood, and showed incomplete haemolysis on horse blood agar. They were highly sensitive to penicillin and to a lesser degree to the cephalosporins and vancomycin; moderately resistant to aminoglycosides; and resistant to sulphonamides and trimethoprim. Synergy was shown between penicillin or vancomycin and streptomycin or gentamicin against all strains. ${ }^{\prime \prime}$

There have been three further reports of Gemella infections, all from France, including a further case of endocarditis ${ }^{12}$ and two cases of septicaemia without endocarditis in patients with alcoholic cirrhosis. ${ }^{1314}$

Meningitis seems to be a rare complication of radiofrequency rhizotomy of the trigeminal ganglion. In a series of 274 patients, submitted to a total of 353 procedures, aseptic meningitis occurred postoperatively in only one patient, who rapidly 
recovered. ${ }^{15}$ In another series of 1200 cases there were only two instances of bacterial meningitis, and both patients recovered rapidly without complications (Professor Jean Siegfried, personal communication). In the present case, the portal of entry of the infecting organism is uncertain. We ascertained during the procedure that the needle did not transfix the buccal mucosa, but it is possible that the organism was already present in the submucosa and was carried as a contaminant up through the foramen ovale.

The present case provides the unique instance of a rare postoperative complication caused by an organism which, at the time of its isolation in 1981, had not previously been reported as a pathogen.

The help of Dr MPE Slack and Dr HH Johnston in establishing a diagnosis is gratefully acknowledged.

\section{References}

' Buchanan RE, Gibbons NE. Bergey's manual of determinative bacteriology. 8th edn. Baltimore: Williams and Wilkins, 1974.

${ }^{2}$ Thjøtta TH, Bøe J. Neisseria haemolysans. A haemolytic species of Neisseria Trevisan. Acta Pathol Microbiol Scand 1938;37 (suppl):527-31.

${ }^{3}$ Berger U, Wezel M. Zur Frage der Identitat hamolysierender saprophytischer Neisserien. Zeitschrift für Hygiene und Infektions Krankheiten. 1960;146:244-52.

${ }^{4}$ Berger U. A proposed new genus of Gram-negative cocci: Gemella. International Bulletin of Bacteriological Nomencla- ture and Taxonomy 1961;11:17-9.

s Yamakawa T, Ueta N. Gas chromatographic studies of microbial components. I. Carbohydrate and fatty acid constitution of Neisseria. Japan J Exp Med 1964;34:361-72.

- Reyn A, Birch-Andersen A, Berger U. Fine structure and taxonomic position of Neisseria haemolysans (Thjøtta and Bøe, 1938) or Gemella haemolysans (Berger, 1960). Acta Pathol Microbiol Scand, Section B 1970;78:375-89.

' Wilkinson BJ, Jones D. A numerical taxonomic survey of Listeria and related bacteria. J Gen Microbiol 1977;98:399-421.

" Stackebrandt E, Wittek B, Seewaldt E, Schleifer KH. Physiological, biochemical and phylogenetic studies on Gemella haemolysans. FEMS Microbiol Lett 1982;13:361-5.

" Facklam RR. Physiological differentiation of viridans streptococci. J Clin Microbiol 1977;5:184-201.

${ }^{10}$ Chatelain R, Croize J, Rouge P, et al. Isolement de Gemella haemolysans dans trois cas d'endocardites bactériennes. Med Mal Infect 1982;12:25-30.

" Buu-Hoi A, Sapoetra A, Branger C, Acar JF. Antimicrobial susceptibility of Gemella haemolysans isolated from patients with or subacute endocarditis. Eur J Clin Microbiol 1982;1:102-6.

${ }^{12}$ Laudat P, Cosnay P, Icole B, Raoult A, Raynaud P, Brochier M. Endocardite à Gemella haemolysans: une nouvelle observation. Medecine et Maladies Infectieuses 1984;14:159-61.

${ }^{13}$ Carles-Giraud D, Dellamonica P, Monnier B, Duplay H. Septicémie à Gemella hemolysans. A propos d'une observation. Méd Mal Infect 1982;12:255-6.

${ }^{14}$ Blin C, Vialette V, Tenaillon A, Fischer D, Cosson G. Sépticemie à Gemella haemolysans. Med Mal Infect 1984; 14: 163-5.

is Sweet WH, Wepsic JG. Controlled thermocoagulation of trigeminal ganglion and rootlets for differential destruction of pain fibres. Part I: trigeminal neuralgia. J Neurosurg 1974;39: 143-56. 